\title{
Implementation of Beyond-Use Date Guidelines for Single-Use Vials at a Pediatric Hospital
}

\author{
Kathryn Hollis, Florence Payen, and Régis Vaillancourt
}

Can J Hosp Pharm. 2021;74(1):70-4

\section{INTRODUCTION}

In September 2016, the National Association of Pharmacy Regulatory Authorities (NAPRA) published the Model Standards for Pharmacy Compounding of Non-hazardous Sterile Preparations ${ }^{1}$ and the Model Standards for Pharmacy Compounding of Hazardous Sterile Preparations. ${ }^{2}$ These documents introduced updated guidelines for establishing beyond-use dates (BUDs) for compounded sterile preparations. In addition to establishing BUDs for the preparations themselves, the guidelines stipulate the length of time that commercially available products used to compound a sterile preparation (either hazardous or nonhazardous) may be used following needle puncture. According to these updated guidelines, the BUD for commercially available single-use vials is $6 \mathrm{~h}$ after needle puncture, if kept in a primary engineering control with ISO Class 5 air quality. If, following needle puncture, the single-use vial is removed from the ISO Class 5 primary engineering control, it must be discarded. Furthermore, if a single-use vial is punctured or opened in an environment with air quality lower than ISO Class 5 , the vial must be discarded after $1 \mathrm{~h}$. The BUD for multiple-dose containers, which typically contain a preservative, is 28 days or the manufacturer's expiry date.

These updated BUD guidelines have important implications for compounding practices in hospital pharmacies because of the potential increase in medication wastage, which will occur if the 6-h BUD is reached before all of the vial contents are used. The Pharmacy Department at the Children's Hospital of Eastern Ontario (CHEO), in Ottawa, Ontario, conducted a study to evaluate medication wastage due to reaching the BUD for single-use vials as set out in the 2016 NAPRA model standards. The ultimate goal was to find a way to reduce waste and the costs associated with such waste. CHEO is a 167-bed pediatric hospital. Its Pharmacy Department is staffed by 19.6 full-time equivalent (FTE) pharmacy technicians and 16.7 FTE pharmacists. Each year, an average of 400000 sterile preparations are compounded by the hospital's pharmacy staff.
The study was designed to determine actual wastage for a 1-week period under different conditions and then to extrapolate from these data to predict wastage over longer periods (1 month and 1 year). The overall study consisted of 3 wastage studies, each lasting 1 week, as described below. The 1-week duration was set in part because of operational and time constraints, but was also intended to capture typical workflows during the week and on the weekend, to reduce potential bias. Results from the 3 wastage studies were then compared with mathematically predicted wastage month-over-month on the basis of vials discarded from August 2018 to July 2019.

The protocol for the study described in this article was reviewed and approved by the Research Ethics Board of the CHEO Research Institute.

\section{EVALUATION OF WASTAGE: ACTUAL AND EXTRAPOLATED VOLUMES OF WASTE}

\section{Wastage Study 1: Volume of Waste before Implementation of NAPRA 2016 BUD Guidelines}

The first step of the evaluation was to document the cost of wastage produced in the Class 5 clean room of the CHEO pharmacy before implementation of the 2016 BUD guidelines. ${ }^{1,2}$ The pharmacy's practice before implementation of the new guidelines was to discard single-use vials when either the volume remaining was insufficient to prepare a complete dose or the vial had expired (according to the manufacturer's guidelines). To determine overall wastage in terms of volume and cost, we collected from the clean room, over a 1-week period, every single-use vial and all compounded medication discarded for any reason. Over this 1-week period, the volume of medication remaining in each discarded single-use vial was completely extracted and measured using syringes (Table 1: volume wasted per week, before implementation of 6-h BUD). The cost of each discarded medication in single-use vials was obtained from pharmacy records. The cost of discarded compounded medications (per millilitre of reconstituted solution) was 


\begin{tabular}{|c|c|c|c|c|c|c|}
\hline \multirow[b]{2}{*}{ Wastage Study } & \multicolumn{3}{|c|}{ Period; Volume Wasted (mL) ${ }^{\mathrm{a}}$} & \multicolumn{3}{|c|}{ Period; Cost of Wastage (\$CAD) ${ }^{b}$} \\
\hline & $\begin{array}{l}\text { Per Week } \\
\text { (Actual) }\end{array}$ & $\begin{array}{c}\text { Per Month } \\
\text { (Extrapolated) }\end{array}$ & $\begin{array}{c}\text { Per Year } \\
\text { (Extrapolated) }\end{array}$ & $\begin{array}{c}\text { Per Week } \\
\text { (Actual) }\end{array}$ & $\begin{array}{c}\text { Per Month } \\
\text { (Extrapolated) }\end{array}$ & $\begin{array}{c}\text { Per Year } \\
\text { (Extrapolated) }\end{array}$ \\
\hline 1: Before implementation of 6-h BUD & 597 & 2602 & 31226 & 399 & 1595 & 20734 \\
\hline 2: After implementation of 6-h BUD & 1317 & 5740 & 68875 & 1355 & 5420 & 70456 \\
\hline $\begin{array}{l}\text { 3: After optimization of use of } \\
\text { different-size vials with 6-h BUD }\end{array}$ & 1110 & 4836 & 58026 & 692 & 2768 & 35987 \\
\hline
\end{tabular}

BUD $=$ beyond-use date.

a Volume of waste was rounded to the nearest millilitre.

${ }^{\mathrm{b}}$ Cost of waste was rounded to the nearest dollar.

calculated using the compounding prices available within the hospital's electronic medical record software. For each drug and concentration, the total volume wasted or lost during the week was calculated and multiplied by the determined price per millilitre. For the list of drugs used in Wastage Study 1, see Appendix 1 (available from cjhp-online.ca/ index.php/cjhp/issue/view/202). The cost of wastage was then summed across all drugs to obtain the total cost of wastage during the week (Table 1: cost of wastage per week, before implementation of 6-h BUD).

\section{Wastage Study 2: Volume of Waste after Implementation of NAPRA 2016 BUD Guidelines}

The next step was to implement the new 6-h BUD guidelines. A label with the BUD (i.e., the date and time at which the vial was to be discarded) was added to each vial when it was punctured or reconstituted. After reconstitution in the primary engineering control, single-use vials were discarded after $6 \mathrm{~h}$. Following implementation of the updated BUD guidelines, Wastage Study 2 was completed according to the methods described above for Wastage Study 1. Most of the vials collected during the 1-week period of Wastage Study 2 were antibiotics (for the list of drugs used in Wastage Study 2, see Appendix 2, available from cjhp-online.ca/ index.php/cjhp/issue/view/202), which had to be reconstituted 3 times a day in order to comply with the new BUD guidelines.

\section{Wastage Study 3: Volume of Waste after Vial-Size Optimization}

Wastage Study 3 was undertaken to optimize the use of different vial sizes (as available from the manufacturer) to reduce waste and maximize the number of doses that could be reconstituted and administered before the BUD limits were reached. For each dose of each medication, the total amount of drug to be reconstituted was calculated to determine which size of vial would be most appropriate to use (for the list of drugs used in Wastage Study 3, see Appendix 3, available from cjhp-online.ca/index.php/ cjhp/issue/view/202). For each drug, the different vial sizes available at the hospital were taken into account and the associated prices recorded. The number of smallersize vials required to exceed the price of the larger-size vial was used to decide the total amount of medication to be compounded; this process created a threshold for determining the vial size that should be used when more than one size of vial existed for a given medication. The bigger vial size was used only if the total amount of the drug to be compounded was greater than the specified threshold. An optimal assortment of vials was then used.

For example, cefazolin is available at our hospital in 1-g and $10 \mathrm{-g}$ vials (see Table 2). According to prices obtained from our supplier, we determined that we could buy seven $1-\mathrm{g}$ vials before reaching the cost of a $10 \mathrm{-g}$ vial. Therefore, to reduce waste and associated costs, when $6 \mathrm{~g}$ or less is to be compounded at the same time, the smaller $(1-\mathrm{g})$ vials must be used. When more than $6 \mathrm{~g}$ is to be compounded at the same time, the larger $(10-\mathrm{g})$ vial must be used. That is deducted from the total amount, and the other vials needed are evaluated with the same method. For example, in practice, for a total amount of $12 \mathrm{~g}$, most of the doses will be prepared from a 10-g vial, with the remainder being prepared from two 1-g vials. The vial-size optimization table (Table 2) was explained to the technicians who actually perform sterile compounding at $\mathrm{CHEO}$, and smaller vials were made available in the anteroom of the clean room, such that all vial sizes were easily accessible for the technicians. Following vial-size optimization, a third 1-week wastage study was conducted, the results of which are also presented in Table 1 (volume wasted and cost of wastage per week, after optimization of use of different-size vials).

\section{Comparison of Wastage across Studies}

Table 1 allows comparison of data from the 3 wastage studies, showing first that the mean volume of wastage over 1 week increased upon implementation of the 6-h BUDs, 
TABLE 2. Optimization of Use of Different-Size Vials for Selected Drugs ${ }^{\mathrm{a}}$

\begin{tabular}{|c|c|c|}
\hline Drug & Vial Size $e^{b}$ & $\begin{array}{c}\text { Total Amount of Drug Suitable } \\
\text { for Vial Size }\end{array}$ \\
\hline Acyclovir & $\begin{array}{l}500 \mathrm{mg} \\
1000 \mathrm{mg}\end{array}$ & $\begin{array}{l}\text { For doses } \leq 500 \mathrm{mg} \text {, use } 500-\mathrm{mg} \text { vial } \\
\text { For doses }>500 \mathrm{mg} \text {, use } 1000-\mathrm{mg} \text { vial }\end{array}$ \\
\hline Caspofungin & $\begin{array}{l}50 \mathrm{mg} \\
70 \mathrm{mg}\end{array}$ & $\begin{array}{l}\text { For doses } \leq 50 \mathrm{mg} \text {, use } 50-\mathrm{mg} \text { vial } \\
\text { For doses }>50 \mathrm{mg} \text {, use } 70-\mathrm{mg} \text { vial }\end{array}$ \\
\hline Cefazolin & $\begin{array}{c}1 \mathrm{~g} \\
10 \mathrm{~g}\end{array}$ & $\begin{array}{l}\text { For doses } \leq 6 \mathrm{~g} \text {, use } 1-\mathrm{g} \text { vials } \\
\text { For doses }>6 \mathrm{~g} \text {, use } 10 \mathrm{~g} \text { vial }\end{array}$ \\
\hline Ceftazidime & $\begin{array}{l}2 g \\
6 g\end{array}$ & $\begin{array}{l}\text { For doses } \leq 4 \mathrm{~g} \text {, use } 2-\mathrm{g} \text { vial } \\
\text { For doses }>4 \mathrm{~g} \text {, use } 6-\mathrm{g} \text { vial }\end{array}$ \\
\hline Ceftriaxone & $\begin{array}{c}1 \mathrm{~g} \\
10 \mathrm{~g}\end{array}$ & $\begin{array}{l}\text { For doses } \leq 10 \mathrm{~g} \text {, use } 1-\mathrm{g} \text { vial } \\
\text { For doses }>10 \mathrm{~g} \text {, use } 10-\mathrm{g} \text { vial }\end{array}$ \\
\hline Cefuroxime & $\begin{array}{l}1.5 \mathrm{~g} \\
7.5 \mathrm{~g}\end{array}$ & $\begin{array}{l}\text { For doses } \leq 7.5 \mathrm{~g} \text {, use } 1.5-\mathrm{g} \text { vial } \\
\text { For doses }>7.5 \mathrm{~g} \text {, use } 7.5 \mathrm{~g} \text { vial }\end{array}$ \\
\hline
\end{tabular}

${ }^{a}$ This table lists only some examples from the complete list of drugs. b This column shows the commercial vial sizes available for each drug. 'Choice of vial size is determined according to the total amount of drug required at the time of drug preparation.

from $597 \mathrm{~mL}$ in Wastage Study 1 to $1317 \mathrm{~mL}$ in Wastage Study 2 . This increase of $720 \mathrm{~mL}$ in weekly wastage represents a $120 \%$ increase in total amount wasted. However, this increase appears to be partially offset by optimization of use of different vial sizes. Specifically, the mean volume wasted in Wastage Study 3 was $1110 \mathrm{~mL}$, which represents only a 513-mL (86\%) increase relative to Wastage Study 1.

What is promising is the apparent $34 \%$ reduction in waste from Wastage Study 2 to Wastage Study 3, following optimization of use of different vial sizes in combination with the new BUD guidelines. It is important to note that implementation of the updated BUD guidelines and vialsize optimization each represent a change to the pharmacy technicians' routine duties. Although the research team was trained on the study protocol, we did not supervise the technicians in the sterile compounding clean room, and we were not able to build the vial optimization protocols into our electronic records. As a result, there may have been technician errors causing wastage (due to the novelty of the procedures and individual technique), as well as changes in workflow relating to the study protocol. Furthermore, it was not possible to optimize utilization of all vial sizes. Some larger vials were used for compounded sterile products despite the recipe requiring a smaller volume. Also, the reconstituted vials used for compounded sterile product recipes were collected, which caused some overestimation of wastage. Taken together, however, these data show that although wastage numbers were still higher under the 2016 BUD parameters, the employment of simple strategies to optimize the use of different vial sizes allowed us to bring actual wastage volumes down by approximately $34 \%$.

\section{ALGORITHMIC WASTAGE PREDICTIONS MONTH-OVER-MONTH AND ASSOCIATED COSTS}

To investigate whether the results from the 3 wastage studies were in agreement with monthly predictions of the volume of wasted drugs, we performed mathematical estimations of the wastage in each vial to generate monthly wastage estimates. Specifically, we used a monthly report produced from the electronic medical record-the Dispense Workload Report-to mathematically predict the volume of wastage and associated costs due to discarded vials from August 2018 to July 2019. The raw data from the reports were submitted to an algorithm in a Visual Basic application (Microsoft Corporation) to calculate the volume of waste from every vial according to the required dose for each drug (Table 3). This process allowed us to calculate the volume wasted and the cost of this wastage for each drug over a 1-year period under 3 different study conditions: before implementation of the 6-h BUD; after implementation of the 6-h BUD; and after vial-size optimization. In the analysis, we considered only dispensed doses directly extracted from vials for the drugs presented in Appendix 3.

The algorithm for wastage calculation before implementation of the 6-h BUD was built on the following assumptions: (1) vials were considered to have been discarded if either the vial's expiry date had passed or the remaining volume was insufficient to prepare a full dose; (2) doses were compounded in the exact order of the data report (i.e., doses dispensed at the same time were not rearranged to reduce the number of vials); (3) when a new vial of a drug was opened, the previous vial of this drug was discarded; (4) the vial size attributed to each drug was always the same; and (5) when the BUD was reached and a dose was required, the dose was delivered before the vial was discarded. The algorithm for calculation of wastage after implementation of the 6-h BUD was built on the basis of the same assumptions, with the exception that the BUD was reduced to $6 \mathrm{~h}$ or less if the manufacturer's BUD was shorter. The algorithm after vialsize optimization used the same assumptions, with the addition of the consideration of vial size. Wasted volumes were slightly overestimated to account for the volume remaining in the vials on the last day of the month; this overestimation is less when the BUD is $6 \mathrm{~h}$.

The performance of the algorithm was assessed by comparing the predicted results with the corresponding extrapolation for the month of the wastage audit. The observed difference in the results for the prediction compared with the extrapolation was likely due to differing work practices of the various pharmacy technicians performing the sterile 
TABLE 3. Algorithmic Prediction of Volume of Wastage and Associated Costs

\begin{tabular}{|c|c|c|c|c|c|c|}
\hline \multirow[b]{2}{*}{ Year and Month } & \multicolumn{3}{|c|}{ Condition; Predicted Volume Wasted (mL) } & \multicolumn{3}{|c|}{ Condition; Cost of Wastage (\$CAD) } \\
\hline & $\begin{array}{l}\text { With Manufacturer } \\
\text { BUD }\end{array}$ & $\begin{array}{c}\text { With } \\
\text { 6-h BUD }\end{array}$ & $\begin{array}{c}\text { With Optimal } \\
\text { Use of Vial Sizes }\end{array}$ & $\begin{array}{l}\text { With Manufacturer } \\
\text { BUD }\end{array}$ & $\begin{array}{c}\text { With } \\
\text { 6-h BUD }\end{array}$ & $\begin{array}{l}\text { With Optimal } \\
\text { Use of Vial Sizes }\end{array}$ \\
\hline \multicolumn{7}{|l|}{2018} \\
\hline August & 20105 & 32627 & 11775 & 6366 & 9870 & 4660 \\
\hline September & 19306 & 32401 & 10026 & 7410 & 11671 & 1966 \\
\hline October & 11951 & 23612 & 10424 & 7908 & 12439 & 1463 \\
\hline November & 13405 & 24963 & 11161 & 7322 & 9933 & 1968 \\
\hline December & 11381 & 22620 & 9487 & 6890 & 9396 & 2661 \\
\hline \multicolumn{7}{|l|}{2019} \\
\hline January & 23810 & 35971 & 13302 & 6799 & 9954 & 3522 \\
\hline February & 11849 & 20798 & 10125 & 4459 & 5960 & 3915 \\
\hline March & 10854 & 23333 & 8655 & 5213 & 7645 & 3798 \\
\hline April & 13746 & 24174 & 10101 & 4507 & 7081 & 5437 \\
\hline May & 18123 & 27964 & 9642 & 5654 & 7725 & 6560 \\
\hline June & 9844 & 22007 & 10842 & 6228 & 8646 & 4515 \\
\hline July & 12376 & 24005 & 10387 & 5813 & 8916 & 4320 \\
\hline Mean & 14729 & 26206 & 10494 & 6214 & 9103 & 3732 \\
\hline SD & 4454 & 4892 & 1196 & 1121 & 1850 & 1517 \\
\hline
\end{tabular}

BUD = beyond-use date, $\mathrm{SD}=$ standard deviation.

compounding (depending on their ability to adapt to new procedures in a short time without any failures of technique). It is important to note that applying vial-size optimization to a greater number of drugs, as well as to recipes for compounded sterile products, would further increase cost savings. Furthermore, although there was significant overlap in the drugs used for the 3 wastage studies reported here (see Appendixes 1,2, and 3, respectively), some drugs appeared in just one study and were absent from the others (e.g., naloxone was considered only in Wastage Study 1, gentamicin was considered only in Wastage Study 2, and caspofungin was considered only in Wastage Study 3). An additional study could be performed with heightened control of the sterile compounding of the drugs, as well as a fixed list of drugs used for both the actual and the algorithmic calculations. Such a study would allow more rigorous comparisons and would improve intertester variability of the wastage measurement, serving to reduce the observed difference between actual and extrapolated values.

In a comparison of predicted wastage according to the manufacturer's BUD with predicted wastage after implementation of the new 6-h BUD, it was determined that the mean predicted total monthly wastage increased from $14729 \mathrm{~mL}$ before implementation of the new 6-h BUD (i.e., manufacturers' BUD) to $26206 \mathrm{~mL}$ after implementation, reflecting an increase in volume of wastage of approximately $78 \%$. In a comparison of predicted wastage according to the manufacturer's BUD with predicted wastage after optimization of use of different vial sizes, the mean predicted total monthly wastage was $10494 \mathrm{~mL}$, a decrease of $29 \%$. Similarly, there was a $60 \%$ decrease in wastage when predicted monthly wastage was compared between the new 6-h BUD and the optimized use of different vial sizes $(26206 \mathrm{~mL}$ and $10494 \mathrm{~mL}$, respectively).

In terms of changes in associated costs (Table 3), the algorithm predicted that the yearly cost of increased wastage caused by implementing the 6-h BUD would be $\$ 34668$ (i.e., mean monthly increase of $\$ 2889 \times 12$ months), a $46 \%$ increase. However, optimization of use of different vial sizes resulted in an estimated yearly saving of $\$ 29784$ (i.e., mean monthly decrease of $\$ 2482 \times 12$ months), despite the need to comply with BUD requirements. These mathematical analyses show that under predicted conditions of optimization of the use of available vial sizes, overall wastage of drugs and resultant costs can be notably reduced month-over-month.

\section{IMPLICATIONS AND SIGNIFICANCE FOR PRACTICE}

Implementation of the updated 6-h BUD guidelines in the central IV admixture clean room of the CHEO pharmacy caused an increase in drug wastage. Specifically, there was a $120 \%$ increase in the volume of wastage measured from Wastage Study 1 to Wastage Study 2. However, by employing simple strategies to optimize use of available vial sizes, we were able to reduce wastage volumes by approximately $34 \%$ (although wastage still remained higher than before implementation of the new BUD guidelines). Furthermore, our algorithm 
predicted that by optimizing the use of different vial sizes, we could generate total savings, year over year, of \$29 784. In our effort to develop cost-reduction methods that are in compliance with good manufacturing practices, we found that an increase in wastage could be mitigated by optimizing the use of various vial sizes. Our prediction models indicate that vial-size optimization will more than offset the additional cost of wastage due to the 6-h BUD. However, in practice, at least when vial-size optimization programs are newly introduced, these savings will likely not be fully realized, since it takes time for staff to become familiar and gain practice with the best way to optimize use of different vial sizes. Reinforcement of optimization practices with technicians, along with continued monitoring, will help in fully realizing cost savings.

\section{References}

1. Model standards for pharmacy compounding of non-hazardous sterile preparations. National Association of Pharmacy Regulatory Authorities; 2016.

2. Model standards for pharmacy compounding of hazardous sterile preparations. National Association of Pharmacy Regulatory Authorities; 2016.
Kathryn Hollis, BScPhm, MHA, is with the Children's Hospital of Eastern Ontario, Ottawa, Ontario.

Florence Payen, BSc, is with the Faculté de Pharmacie de Lyon, Université Claude Bernard Lyon 1, Lyon, France.

Régis Vaillancourt, BScPharm, PharmD, FCSHP, FFIP, is with the Children's Hospital of Eastern Ontario, Ottawa, Ontario.

Competing interests: None declared.

\section{Address correspondence to:}

Kathryn Hollis

Children's Hospital of Eastern Ontario

401 Smyth Road

Ottawa ON K1H 8L1

email: khollis@cheo.on.ca

Funding: None received.

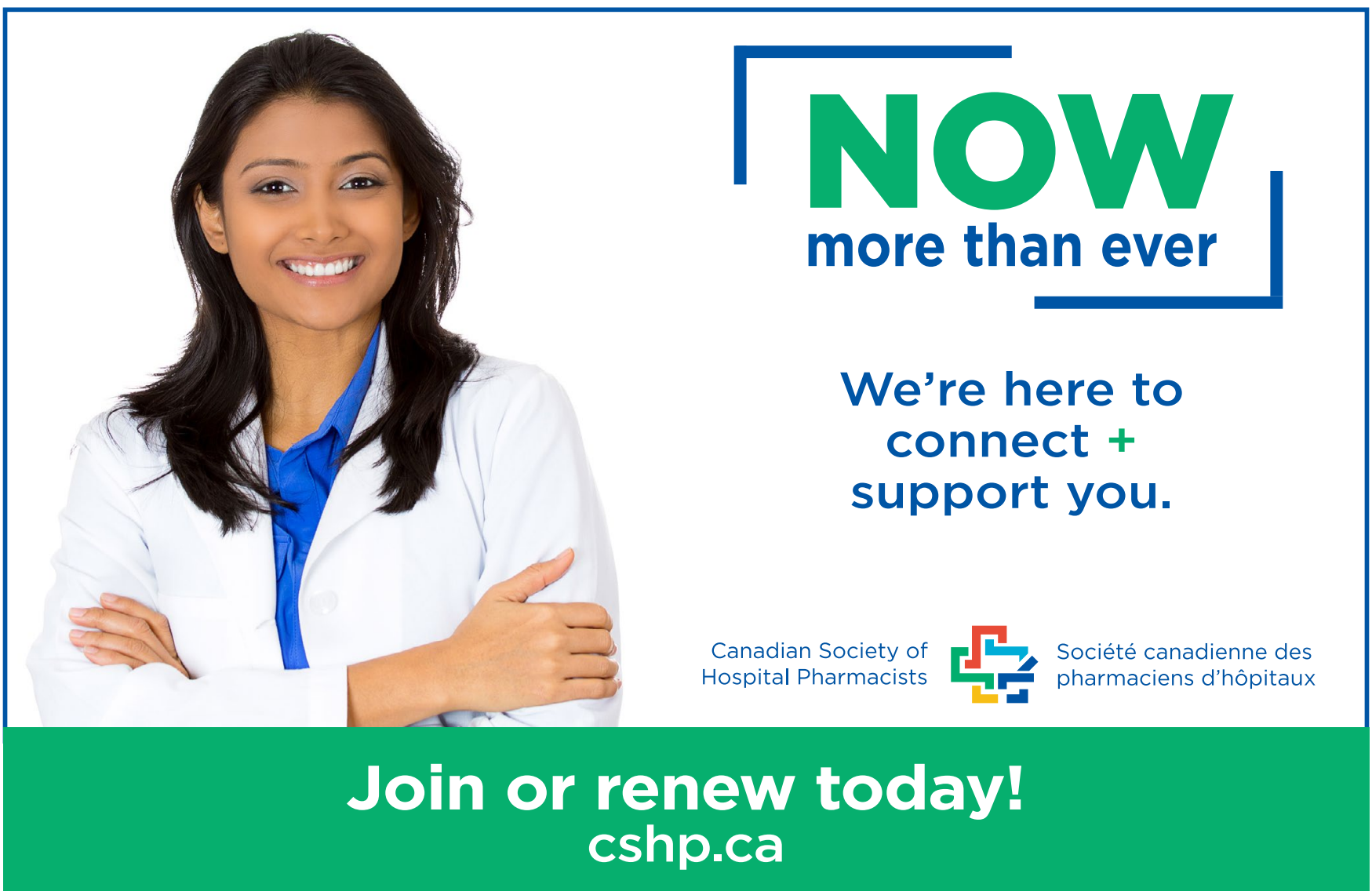

